\title{
A BOUNDARY VALUE PROBLEM FOR AN EQUATION OF MIXED TYPE
}

BY

M. H. PROTTER

1. Introduction. We consider a boundary value problem for the equation

$$
K(y) u_{x x}+u_{y y}=0
$$

where $K(y)$ is a continuous nondecreasing function with $K(0)=0$. Particular cases of (1.1) have been the subject of several investigations. In particular Darboux [4](1), Tricomi [8], and Cibrario [3] have investigated the case $K(y)=y$. Gellerstedt [6] considered the case $K(y)=y^{n}$.

Within the last few years interest in equation (1.1) has been stimulated by the problems of transsonic flow. The equation of Chaplygin for a twodimensional gas flow, when transformed to the hodograph plane, is of the form (1.1) with $K(y)$ positive for subsonic speeds and negative for supersonic speeds. In this connection Frankl [5] investigated initial value problems for an equation of mixed type similar to (1.1). For (1.1) Bers announced an existence and uniqueness theorem for the Dirichlet problem in which the domain lies in the elliptic portion of the plane but has part of its boundary on the parabolic line [1]. He solved the Cauchy problem for a hyperbolic domain with the data prescribed along the parabolic line [2]. Recently Germain and Bader [7] have announced results similar to those of Bers and of the author. The similarities are discussed later in this section.

We consider the boundary value problem for a hyperbolic-parabolic domain in which the data are prescribed along one characteristic and the parabolic line. We prove the following

THEOREM 1.1. Let $F_{0}(x), a \leqq x \leqq b$, and $G_{0}(x),(a+b) / 2 \leqq x \leqq b$, be functions possessing continuous fourth derivatives with $F_{0}(b)=G_{0}(b)$. Let $y=h(x)$ be the equation of the characteristic curve of $(1.1)$ passing through the point $(b, 0)$ having positive slope. Then there exists a unique twice continuously differentiable solution $u(x, y)$ of equation (1.1) in the curvilinear triangular domain bounded by the segment of the $x$-axis $a \leqq x \leqq b$, the characteristic $y=h(x)$, and the symmetrical characteristic through the point $(a, 0)$ which satisfies the conditions $u(x, 0)=F_{0}(x), a \leqq x \leqq b, u[x, h(x)]=G_{0}(x),(a+b) / 2 \leqq x \leqq b$. In this domain

$$
|u(x, y)| \leqq M_{0}+4|y| M_{1} \cdot M_{2}
$$

where $M_{0}=\max \left(\left|F_{0}(x)\right|,\left|G_{0}(x)\right|\right), M_{1}=\max \left(\left|F_{0}^{\prime}(x)\right|,\left|G_{0}^{\prime}(x)\right|\right)$, and $M_{2}$ $=\max (-K(y))^{1 / 2}$.

Presented to the Society, October 29, 1949; received by the editors March 15, 1951

(1) Numbers in brackets refer to the bibliography at the end of the paper. 
Germain and Bader considered, as did Bers, the Cauchy problem for (1.1) with the given data along the parabolic line; they also treated the boundary value problem as stated in Theorem 1.1. However in both problems Germain and Bader impose the additional condition $K(y)=O(|y|)$ as $y \rightarrow 0$.

The Tricomi problem for equation (1.1) is one in which boundary values are assigned along one characteristic and along an arc in the elliptic portion of the plane one of whose end points meets the given characteristic on the parabolic line and whose other end point also lies on the parabolic line. Theorem 1.1 deals with a limiting case of the Tricomi problem in which the arc in the elliptic portion of the plane reduces to a segment along the parabolic line.

2. The step-function case. We consider in this section the equation

$$
K^{*}(y) u_{x x}+u_{y y}=0
$$

where $K^{*}(y)$ is a nondecreasing step-function with $m$ steps:

$$
K^{*}(y)=-\lambda_{i}^{2}, \quad y_{i} \leqq y<y_{i-1}, \quad i=1,2, \cdots, m .
$$

We suppose that $\lambda_{1}^{2}>0, y_{0}=0, y_{m}=c<0$. Let $y=h_{1}(x)$ and $y=h_{2}(x)$ be the equations of the characteristics through the points $(a, 0)$ and $(b, 0)$ respectively which meet at the point $(a / 2+b / 2, c)$. These characteristics are of course polygonal arcs. Let $D(a, b)$ designate the domain bounded by $h_{1}(x)$, $h_{2}(x)$, and the interval $(a, b)$ along the $x$-axis. Without loss of generality we select the interval $(a, b)$ to be the interval $(0,2)$. Noting the fact that $(2.1)$ reduces to the one-dimensional wave equation in each of the strips, we may represent a solution $u(x, y)$ in the form

$$
u(x, y)=f_{i}\left(x+\lambda_{i} y\right)+g_{i}\left(x-\lambda_{i} y\right)
$$

for the strip $y_{i} \leqq y \leqq y_{i-1}, i=1,2, \cdots, m$. A solution of $(2.1)$ is defined as any function satisfying $(2.1)$ for $y \neq y_{i}$ which in $D(0,2)$ has continuous partial derivatives of the second order, except at the boundaries of the $m$ strips where only the partial derivatives of the first order are required to be continuous.

A weak solution of $(2.1)$ is defined as any function $u(x, y)$ which has the form (2.2) in each of the $m$ strips and which on the boundaries of the strips satisfies the conditions

$$
\begin{aligned}
& f_{i+1}\left(x+\lambda_{i+1} y_{i}\right)=\frac{\lambda_{i+1}+\lambda_{i}}{2 \lambda_{i+1}} f_{i}\left(x+\lambda_{i} y_{i}\right)+\frac{\lambda_{i+1}-\lambda_{i}}{2 \lambda_{i+1}} g_{i}\left(x-\lambda_{i} y_{i}\right), \\
& g_{i+1}\left(x-\lambda_{i+1} y_{i}\right)=\frac{\lambda_{i+1}-\lambda_{i}}{2 \lambda_{i+1}} f_{i}\left(x+\lambda_{i} y_{i}\right)+\frac{\lambda_{i+1}+\lambda_{i}}{2 \lambda_{i+1}} g_{i}\left(x-\lambda_{i} y_{i}\right)
\end{aligned}
$$

for $i=1,2, \cdots, m-1$. From equations (2.3) it follows at once that if each of the functions $f_{i}\left(x+\lambda_{i} y\right), g_{i}\left(x-\lambda_{i} y\right), i=1,2, \cdots, m$, is continuously dif- 
ferentiable, then $u(x, y)$ will be continuously differentiable throughout $D(0,2)$. If the $f_{i}$ and $g_{i}$ have continuous partial derivatives of the second order, then a weak solution is a solution.

Lemma 2.1. Let $F_{0}(x)$ be a given differentiable function with $F_{0}(2)=0$. Then there exists a unique weak solution $u(x, y)$ of equation $(2.1)$ in $D(0,2)$ satisfying the conditions $u(x, 0)=F_{0}(x), 0 \leqq x \leqq 2$, and $u\left[x, h_{2}(x)\right]=0,1 \leqq x \leqq 2$. For $y_{1} \leqq y \leqq 0$ the solution $u(x, y)$ may be represented in the form $u(x, y)$ $=f_{1}\left(x+\lambda_{1} y\right)+g_{1}\left(x-\lambda_{1} y\right)$, and the functions $f_{1}, g_{1}$ satisfy the inequalities

$$
\left|f_{1}\left(x+\lambda_{1} y\right)\right| \leqq \frac{\lambda_{m}}{\lambda_{1}} M, \quad\left|g_{1}\left(x-\lambda_{1} y\right)\right| \leqq \frac{\lambda_{m}}{\lambda_{1}} M,
$$

$M=\max \left|F_{0}(x)\right|, 0 \leqq x \leqq 2$.

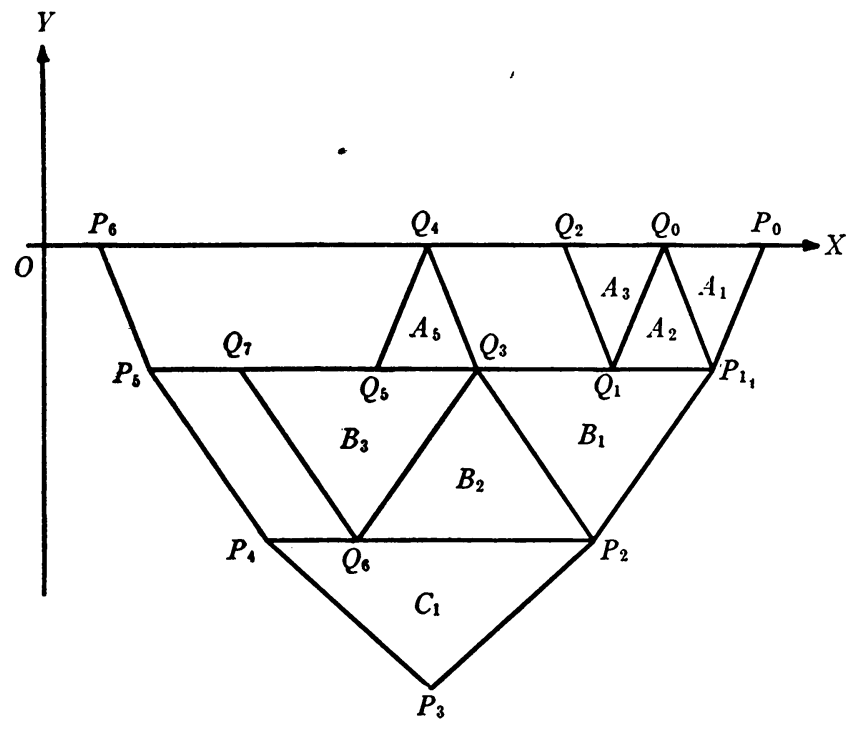

FIG. 1

Proof. The existence and uniqueness will be established by constructing the solution. We proceed by induction. However, to make the process clear the case for three strips $(m=3)$ will first be worked out in detail. Following this we give the induction proof.

Let $A_{1}$ denote the triangle $P_{0} P_{1} Q_{0}$ (see Fig. 1 in which all lines drawn which are not parallel to the $x$-axis are characteristics). In general we shall denote the maximum of the absolute value of a function $\phi$ in an area $A$ by $|\phi|_{A}$. In $A_{1}$ we have as the solution of $(2.1)$ a function $u(x, y)$ of the form $f_{1}\left(x+\lambda_{1} y\right)+g_{1}\left(x-\lambda_{1} y\right)$. For $y_{1} \leqq y \leqq 0$ the characteristic $h_{2}(x)$ is the straight line $x-\lambda_{1} y=2$. Since $u\left[x, h_{2}(x)\right]=0$ we have, if we select $g_{1}(2)=0, f_{1}(2 x-2)$ $=0$ along $h_{2}(x)$. Therefore $f_{1}\left(x+\lambda_{1} y\right)=0$ in $A_{1}$. Thus $u(x, y)$ is reduced to the 
form $g_{1}\left(x-\lambda_{1} y\right)$ in $A_{1}$. From the condition $u(x, 0)=F_{0}(x)$ we see that $g_{1}(x)$ $=F_{0}(x), 2+2 \lambda_{1} y_{1} \leqq x \leqq 2$. Hence the existence of $u(x, y)$ is established throughout $A_{1}$ and we note that $\left|g_{1}\right|_{A_{1}} \leqq M$. Since $g_{1}$ is a function of $x-\lambda_{1} y$ its definition is immediately extended to the triangle $A_{2}: P_{1} Q_{0} Q_{1}$ and we have $\left|g_{1}\right|_{A_{2}} \leqq M$. We proceed now to the triangle $B_{1}: P_{1} P_{2} Q_{3}$. In $B_{1}$ the solution $u(x, y)$ is of the form $f_{2}\left(x+\lambda_{2} y\right)+g_{2}\left(x-\lambda_{2} y\right)$. By the same reasoning employed for $A_{1}$ we know $f_{2}\left(x+\lambda_{2} y\right)$ throughout $B_{1}$. Employing equations (2.3) and taking into account the fact that $f_{2}\left(x+\lambda_{2} y\right)=0$ along the segment $P_{1} Q_{3}$, we have along this segment

$$
\begin{aligned}
& g_{2}\left(x-\lambda_{2} y_{1}\right)=\frac{2 \lambda_{1}}{\lambda_{1}+\lambda_{2}} g_{1}\left(x-\lambda_{1} y_{1}\right), \\
& f_{1}\left(x+\lambda_{1} y_{1}\right)=-\frac{\lambda_{2}-\lambda_{1}}{\lambda_{2}+\lambda_{1}} g_{1}\left(x-\lambda_{1} y_{1}\right) .
\end{aligned}
$$

Equation (2.5) defines $g_{2}\left(x-\lambda_{2} y\right)$ for $B_{1}$ and (2.6) defines $f_{1}\left(x+\lambda_{1} y\right)$ for $A_{2}$. Thus the solution is determined completely in $A_{1}, A_{2}$, and $B_{1}$. From the fact that $f_{1}$ is a function of $x+\lambda_{1} y$ its definition is extended from $A_{2}$ to $A_{3}: Q_{0} Q_{1} Q_{2}$. In triangle $A_{3}$ we have for $y=0, u(x, 0)=f_{1}(x)+g_{1}(x)=F_{0}(x), 2+4 \lambda_{1} y_{1} \leqq x$ $\leqq 2+2 \lambda_{1} y_{1}$. This determines $g_{1}(x)$ along the segment $Q_{0} Q_{2}$ and hence in $A_{3}$ and $A_{4}$ (not shown in Fig. 1). The solution $u$ is now established in $A_{3}$. From equation (2.6) we have

$$
f_{1}(x)=-\frac{\lambda_{2}-\lambda_{1}}{\lambda_{2}+\lambda_{1}} g_{1}\left(x-2 \lambda_{1} y_{1}\right)
$$

which implies that

$$
\left|f_{1}\right|_{A_{3}} \leqq \frac{\lambda_{2}-\lambda_{1}}{\lambda_{2}+\lambda_{1}}\left|g_{1}\right|_{A_{1}} \leqq \frac{\lambda_{2}-\lambda_{1}}{\lambda_{2}+\lambda_{1}} M .
$$

For the estimate in $A_{3}$ we find

$$
\begin{aligned}
\left|g_{1}\right|_{A_{3}} & \leqq\left|F_{0}\right|_{A_{3}}+\frac{\lambda_{2}-\lambda_{1}}{\lambda_{2}+\lambda_{1}}\left|g_{1}\right|_{A_{1}} \leqq M\left(1+\frac{\lambda_{2}-\lambda_{1}}{\lambda_{2}+\lambda_{1}}\right) \\
& =\frac{2 \lambda_{2}}{\lambda_{1}+\lambda_{2}} M \leqq \frac{\lambda_{1}+\lambda_{2}}{2 \lambda_{1}} M .
\end{aligned}
$$

Proceeding to the succeeding triangles we have for all triangles in $P_{0} P_{1} Q_{3} Q_{4}$

$$
\begin{aligned}
& \left|f_{1}\right|_{A_{j}} \leqq \frac{\lambda_{2}-\lambda_{1}}{\lambda_{2}+\lambda_{1}}\left|g_{1}\right|_{A_{j-2}}=M \frac{\lambda_{2}-\lambda_{1}}{\lambda_{2}+\lambda_{1}} \cdot \frac{\lambda_{2}+\lambda_{1}}{2 \lambda_{1}}=\frac{\lambda_{2}-\lambda_{1}}{2 \lambda_{1}} M, \\
& \left|g_{1}\right|_{A_{j}} \leqq \frac{\lambda_{2}+\lambda_{1}}{2 \lambda_{1}} M .
\end{aligned}
$$


We now wish to establish the solution and obtain estimates in the triangle $A_{5}: Q_{3} Q_{4} Q_{5} . g_{1}\left(x-\lambda_{1} y\right)$ is established in $A_{5}$ from its determination in the trapezoid $P_{0} P_{1} Q_{3} Q_{4}$. Along the segment $Q_{3} Q_{5}$ we have

$$
f_{1}\left(x+\lambda_{1} y_{1}\right)=\frac{2 \lambda_{2}}{\lambda_{2}+\lambda_{1}} f_{2}\left(x+\lambda_{2} y_{1}\right)-\frac{\lambda_{2}-\lambda_{1}}{\lambda_{2}+\lambda_{1}} g_{1}\left(x-\lambda_{1} y_{1}\right) .
$$

To determine $f_{1}\left(x+\lambda_{1} y\right)$ in $A_{5}$ it is necessary only to obtain $f_{2}\left(x+\lambda_{2} y\right)$ in $B_{3}: Q_{3} Q_{6} Q_{7}$. For this purpose we find $g_{2}$ and $f_{2}$ in $B_{2}: P_{2} Q_{3} Q_{6}$. In $B_{2}$ the function $g_{2}$ is known from its values in $B_{1}$. Proceeding now to $C_{1}: P_{2} P_{3} P_{4}$ we see that $f_{3}\left(x+\lambda_{3} y\right)=0$ and therefore from (2.3)

$$
f_{2}\left(x+\lambda_{2} y_{2}\right)=-\frac{\lambda_{3}-\lambda_{2}}{\lambda_{3}+\lambda_{2}} g_{2}\left(x-\lambda_{2} y_{2}\right) .
$$

Hence $u(x, y)$ is determined in $B_{2}$ and from this $f_{2}\left(x+\lambda_{2} y\right)$ is defined in $B_{3}$. From (2.8) we now have $f_{1}\left(x+\lambda_{1} y\right)$ in $A_{5}$. To obtain a bound for $f_{1}$ in $A_{5}$ we have from (2.8)

$$
\left|f_{1}\right|_{A_{5}} \leqq \frac{2 \lambda_{2}}{\lambda_{2}+\lambda_{1}}\left|f_{2}\right|_{B_{3}}+\frac{\lambda_{2}-\lambda_{1}}{\lambda_{2}+\lambda_{1}}\left|g_{1}\right|_{A_{5}} .
$$

Also, from (2.7) we have $\left|g_{1}\right|_{A_{5}} \leqq\left(\left(\lambda_{2}+\lambda_{1}\right) / 2 \lambda_{1}\right) M$. Combining (2.9) and (2.5) we get

$$
\left|f_{2}\right|_{B_{3}} \leqq \frac{\lambda_{3}-\lambda_{2}}{\lambda_{3}+\lambda_{2}}\left|g_{2}\right|_{B_{1}} \leqq \frac{\lambda_{3}-\lambda_{2}}{\lambda_{3}+\lambda_{2}} \cdot \frac{2 \lambda_{1}}{\lambda_{2}+\lambda_{1}}\left|g_{1}\right|_{P_{0} P_{1} Q_{3} Q_{4}},
$$

and taking (2.7) into account we obtain

$$
\left|f_{2}\right|_{B_{3}} \leqq \frac{\lambda_{3}-\lambda_{2}}{\lambda_{3}+\lambda_{2}} M
$$

These inequalities yield

$$
\left|f_{1}\right|_{A_{5}} \leqq \frac{2 \lambda_{2}}{\lambda_{2}+\lambda_{1}} \cdot \frac{\lambda_{3}-\lambda_{2}}{\lambda_{3}+\lambda_{2}} M+\frac{\lambda_{2}-\lambda_{1}}{\lambda_{2}+\lambda_{1}} \cdot \frac{\lambda_{2}+\lambda_{1}}{2 \lambda_{1}} M \leqq \frac{\lambda_{3}-\lambda_{1}}{2 \lambda_{1}} M .
$$

Continuing in this way we obtain the solution throughout the parallelogram: $Q_{4} Q_{3} P_{5} P_{6}$, and in this parallelogram we have the bounds

$$
\left|f_{1}\right| \leqq \frac{\lambda_{3}-\lambda_{1}}{2 \lambda_{1}} M \leqq \frac{\lambda_{3}}{\lambda_{1}} M, \quad\left|g_{1}\right| \leqq \frac{\lambda_{3}+\lambda_{1}}{2 \lambda_{1}} M \leqq \frac{\lambda_{3}}{\lambda_{1}} M .
$$

For the general case we suppose that we have established the solution and the inequalities as shown above for the first $n-1$ of the $m$ strips. We wish to extend the solution and the inequalities to the domain involving the $n$th 
strip. (See Fig. 2 for which $n=5$.) For triangle $T_{1}: S_{1} S_{2} S_{3}$ we have obtained $g_{1}\left(x+\lambda_{1} y\right)$ from the results for $n-1$ strips and also the estimate $\left|g_{1}\right| r_{1}$ $\leqq\left(\lambda_{n-1}+\lambda_{1}\right) / 2 \lambda_{1}$. From (2.3) we have along the segment $S_{2} S_{3}$ :

$$
f_{1}\left(x+\lambda_{1} y_{1}\right)=\frac{2 \lambda_{2}}{\lambda_{2}+\lambda_{1}} f_{2}\left(x+\lambda_{2} y_{1}\right)+\frac{\lambda_{2}-\lambda_{1}}{\lambda_{2}+\lambda_{1}} g_{1}\left(x-\lambda_{1} y_{1}\right) .
$$

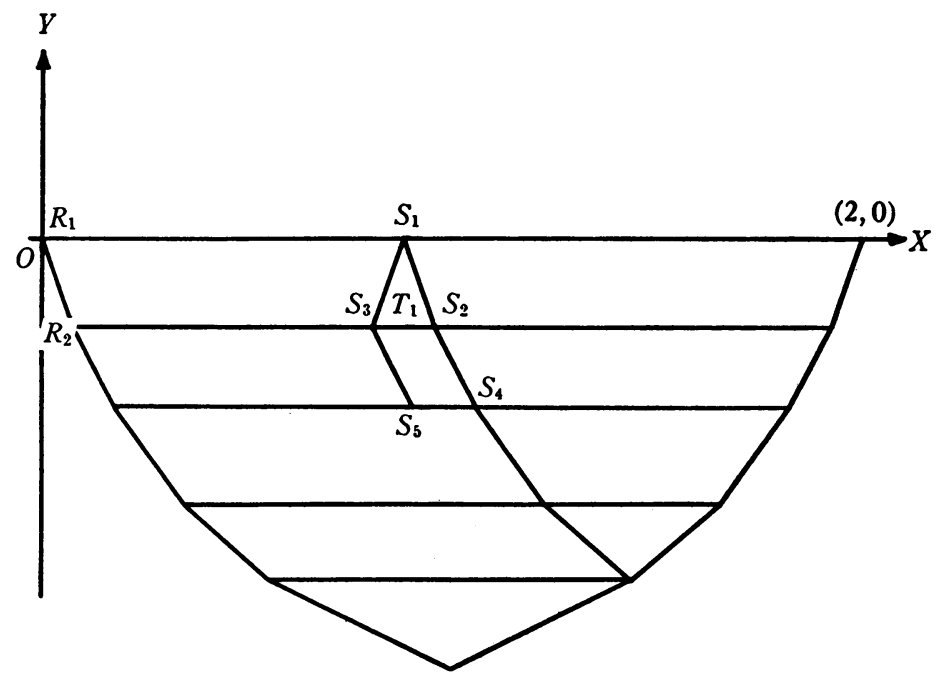

FIG. 2

Since $g_{1}$ is already estimated we must find and estimate only $f_{2}\left(x+\lambda_{2} y\right)$ along $S_{2} S_{3}$. Noticing that $f_{2}\left(x+\lambda_{2} y\right)$ along $S_{2} S_{3}$ is the same as $f_{2}\left(x+\lambda_{2} y\right)$ along $S_{4} S_{5}$, we have the equation

$$
f_{2}\left(x+\lambda_{2} y_{2}\right)=\frac{2 \lambda_{3}}{\lambda_{3}+\lambda_{2}} f_{3}\left(x+\lambda_{3} y_{2}\right)-\frac{\lambda_{3}-\lambda_{2}}{\lambda_{3}+\lambda_{2}} g_{2}\left(x-\lambda_{2} y_{2}\right)
$$

and the inequality

$$
\left|f_{2}\right|_{S_{2} S_{3}} \leqq \frac{2 \lambda_{3}}{\lambda_{3}+\lambda_{2}}\left|f_{3}\right|_{S_{4} S_{5}}+\frac{\lambda_{3}-\lambda_{2}}{\lambda_{3}+\lambda_{2}}\left|g_{2}\right|_{S_{4} S_{5}}
$$

$g_{2}$ has already been determined and estimated from the $n-1$ strip case and found to be dominated by $\left(\left(\lambda_{n-1}+\lambda_{2}\right) / 2 \lambda_{2}\right) M$. Therefore we must find and estimate $f_{3}\left(x+\lambda_{3} y\right)$ along $S_{4} S_{5}$. Proceeding in this way we are led to the $n$th strip. However, here we have $f_{n}\left(x+\lambda_{n} y\right)=0$. Then from (2.3) we have

$$
f_{n-1}\left(x+\lambda_{n-1} y_{n-1}\right)=-\frac{\lambda_{n}-\lambda_{n-1}}{\lambda_{n}+\lambda_{n-1}} g_{n-1}\left(x-\lambda_{n-1} y_{n-1}\right),
$$




$$
\left|f_{n-1}\right| \leqq \frac{\lambda_{n}-\lambda_{n-1}}{\lambda_{n}+\lambda_{n-1}} \cdot \frac{\lambda_{n}+\lambda_{n-1}}{2 \lambda_{n-1}} M=\frac{\lambda_{n}-\lambda_{n-1}}{2 \lambda_{n-1}} M .
$$

Proceeding now in the other direction we find

$$
\begin{aligned}
f_{n-2}\left(x+\lambda_{n-2} y_{n-2}\right)= & \frac{2 \lambda_{n-1}}{\lambda_{n-1}+\lambda_{n-2}} f_{n-1}\left(x+\lambda_{n-1} y_{n-2}\right) \\
& -\frac{\lambda_{n-1}-\lambda_{n-2}}{\lambda_{n-1}+\lambda_{n-2}} g_{n-2}\left(x-\lambda_{n-2} y_{n-2}\right)
\end{aligned}
$$

and

$$
\left|f_{n-2}\right| \leqq \frac{2 \lambda_{n-1}}{\lambda_{n-1}+\lambda_{n-2}}\left|f_{n-1}\right|+\frac{\lambda_{n-1}-\lambda_{n-2}}{\lambda_{n-1}+\lambda_{n-2}}\left|g_{n-2}\right| \leqq \frac{\lambda_{n}-\lambda_{n-2}}{2 \lambda_{n-2}} M .
$$

Continuing, we finally obtain $f_{1}\left(x+\lambda_{1} y\right)$ and get the inequality

$$
\left|f_{1}\right| \leqq \frac{\lambda_{n}-\lambda_{1}}{2 \lambda_{1}} M
$$

This yields the solution throughout the parallelogram $S_{1} S_{2} R_{2} R_{1}$ and the estimates

$$
\left|f_{1}\right| \leqq \frac{\lambda_{n}-\lambda_{1}}{2 \lambda_{1}} M \leqq \frac{\lambda_{n}}{\lambda_{1}} M, \quad\left|g_{1}\right| \leqq \frac{\lambda_{n}+\lambda_{1}}{2 \lambda_{1}} M \leqq \frac{\lambda_{n}}{\lambda_{1}} M .
$$

Lemma 2.2. Let $G_{0}(x)$ be a given differentiable function with $G_{0}(2)=0$. Then there exists a unique weak solution $u(x, y)$ of equation $(2.1)$ in $D(0,2)$ satisfying the conditions $u(x, 0)=0,0 \leqq x \leqq 2$, and $u\left[x, h_{2}(x)\right]=G_{0}(x), 1 \leqq x \leqq 2$. For $y_{1} \leqq y$ $\leqq 0$ the solution $u(x, y)$ may be represented in the form $u(x, y)=f_{1}\left(x+\lambda_{1} y\right)$ $+g_{1}\left(x-\lambda_{1} y\right)$, and the functions $f_{1}\left(x+\lambda_{1} y\right), g_{1}\left(x-\lambda_{1} y\right)$ satisfy the inequalities

$$
\left|f_{1}\right| \leqq \frac{\lambda_{m}}{\lambda_{1}} M, \quad\left|g_{1}\right| \leqq \frac{\lambda_{m}}{\lambda_{1}} M,
$$

$M=\max \left|G_{0}(x)\right|, 1 \leqq x \leqq 2$.

Proof. We proceed as in Lemma 2.1. Referring to Fig. 1 we have in triangle $A_{1}, u(x, y)=f_{1}\left(x+\lambda_{1} y\right)+g_{1}\left(x-\lambda_{1} y\right)$. Without loss of generality we may select $g_{1}(2)=0$; then $f_{1}(2 x-2)=G_{0}(x), 2+\lambda_{1} y \leqq x \leqq 2$. Thus in $A_{1},\left|f_{1}\right|$ $\leqq M$. By hypothesis $f_{1}(x)+g_{1}(x)=0,2+2 \lambda_{1} y_{1} \leqq x \leqq 2$, and therefore $g_{1}\left(x-\lambda_{1} y\right)$ and hence $u(x, y)$ is completely determined in $A_{1}$. In addition, $\left|g_{1}\right|_{A_{1}} \leqq M$ and consequently $\left|g_{1}\right|_{A_{2}} \leqq M$. Employing (2.3) we find along $P_{1} Q_{3}$

$$
f_{1}\left(x+\lambda_{1} y_{1}\right)=\frac{2 \lambda_{2}}{\lambda_{2}+\lambda_{1}} f_{2}\left(x+\lambda_{2} y_{1}\right)+\frac{\lambda_{1}-\lambda_{2}}{\lambda_{1}+\lambda_{2}} g_{1}\left(x-\lambda_{1} y_{1}\right)
$$


where we have taken $u(x, y)$ to be of the form $f_{2}\left(x+\lambda_{2} y\right)+g_{2}\left(x-\lambda_{2} y\right)$ in the second strip. The function $f_{2}\left(x+\lambda_{2} y\right)$ is determined in $B_{1}$ by $G_{0}(x)$ and hence $\left|f_{2}\right|_{B_{1}} \leqq M$. Thus

$$
\left|f_{1}\right|_{A_{2}} \leqq \frac{2 \lambda_{2}}{\lambda_{2}+\lambda_{1}} M+\frac{\lambda_{2}-\lambda_{1}}{\lambda_{2}+\lambda_{1}} M \leqq \frac{\lambda_{2}}{\lambda_{1}} M .
$$

Again employing the relation $f_{1}(x)+g_{1}(x)=0$, we find $\left|g_{1}\right|_{A_{3}} \leqq \lambda_{2} M / \lambda_{1}$, $\left|g_{1}\right|_{A_{4}} \leqq \lambda_{2} M / \lambda_{1}$, and therefore

$$
\left|f_{1}\right|_{A_{4}} \leqq \frac{2 \lambda_{2}}{\lambda_{2}+\lambda_{1}} M+\frac{\lambda_{2}-\lambda_{1}}{\lambda_{2}+\lambda_{1}} \cdot \frac{\lambda_{2}}{\lambda_{1}} M=\frac{\lambda_{2}}{\lambda_{1}} M .
$$

Thus we have in the trapezoid $P_{0} P_{1} Q_{3} Q_{4}$

$$
\left|g_{1}\right| \leqq \frac{\lambda_{2}}{\lambda_{1}} M, \quad\left|f_{1}\right| \leqq \frac{\lambda_{2}}{\lambda_{1}} M .
$$

To continue the process we determine $g_{2}\left(x-\lambda_{2} y\right)$ in $B_{1}$ from the relation

$$
g_{2}\left(x-\lambda_{2} y_{1}\right)=\frac{2 \lambda_{1}}{\lambda_{2}+\lambda_{1}} g_{1}\left(x-\lambda_{1} y_{1}\right)+\frac{\lambda_{2}-\lambda_{1}}{\lambda_{2}+\lambda_{1}} f_{2}\left(x+\lambda_{2} y_{1}\right)
$$

and we get the estimate

$$
\left|g_{2}\right| \leqq \frac{2 \lambda_{2}}{\lambda_{2}+\lambda_{1}} M+\frac{\lambda_{2}-\lambda_{1}}{\lambda_{2}+\lambda_{1}} M \leqq \frac{\lambda_{2}}{\lambda_{1}} M
$$

which is valid in $B_{1}$ and consequently in $B_{2}$. In $C_{1}$ we take $u(x, y)=f_{3}\left(x+\lambda_{3} y\right)$ $+g_{3}\left(x-\lambda_{3} y\right)$ and since $f_{3}\left(x+\lambda_{3} y\right)=G_{0}(x)$ along the characteristic $P_{2} P_{3}$, $f_{3}\left(x+\lambda_{3} y\right)$ is defined throughout $C_{1}$; also $\left|f_{3}\right| c_{1} \leqq M$. The definition of $g_{1}\left(x-\lambda_{1} y\right)$ is extended to $A_{5}$ from its definition in the trapezoid $P_{0} P_{1} Q_{3} Q_{4}$; therefore $\left|g_{1}\right|_{A_{5}} \leqq \lambda_{2} M / \lambda_{1}$. To estimate $f_{1}$ in $A_{5}$ we must estimate $f_{2}\left(x+\lambda_{2} y\right)$ along $Q_{3} Q_{5}$ and employ (2.8). For this purpose we use the relation

$$
f_{2}\left(x+\lambda_{2} y_{2}\right)=\frac{2 \lambda_{3}}{\lambda_{3}+\lambda_{2}} f_{3}\left(x+\lambda_{3} y_{2}\right)-\frac{\lambda_{3}-\lambda_{2}}{\lambda_{3}+\lambda_{2}} g_{2}\left(x-\lambda_{2} y_{2}\right)
$$

which is valid in $B_{2}$. We now have the estimate for $f_{1}$ in $A_{5}$ :

$$
\left|f_{1}\right|_{A_{5}} \leqq\left\{\frac{2 \lambda_{2}}{\lambda_{2}+\lambda_{1}}\left[\frac{2 \lambda_{3}}{\lambda_{3}+\lambda_{2}}+\frac{\lambda_{3}-\lambda_{2}}{\lambda_{3}+\lambda_{2}} \cdot \frac{\lambda_{2}}{\lambda_{1}}\right]+\frac{\lambda_{2}-\lambda_{1}}{\lambda_{2}+\lambda_{1}} \cdot \frac{\lambda_{2}}{\lambda_{1}}\right\} M .
$$

From this it is easily verified that $\left|f_{1}\right|_{A_{5}} \leqq \lambda_{3} M / \lambda_{1}$. The argument for the general case then proceeds as in Lemma 2.1.

Because of the linearity of the problem Lemmas 2.1 and 2.2 may be combined in the following lemma. 
Lemma 2.3. Let $F_{0}(x), 0 \leqq x \leqq 2, G_{0}(x), 1 \leqq x \leqq 2$, be differentiable functions with $F_{0}(2)=G_{0}(2)$. Then there exists a unique weak solution of equation (2.1) in $D(0,2)$ satisfying the conditions $u(x, 0)=F_{0}(x), 0 \leqq x \leqq 2, u\left[x, h_{2}(x)\right]=G_{0}(x)$, $1 \leqq x \leqq 2$. In the strip $y_{1} \leqq y \leqq 0, u(x, y)$ may be represented in the form $u(x, y)$ $=f_{1}\left(x+\lambda_{1} y\right)+g_{1}\left(x-\lambda_{1} y\right)$ and in this strip $f_{1}$ and $g_{1}$ satisfy the inequalities

$$
\left|f_{1}\right| \leqq \frac{2 \lambda_{m}}{\lambda_{1}} M, \quad\left|g_{1}\right| \leqq \frac{2 \lambda_{m}}{\lambda_{1}} M,
$$

$M=\max \left(\left|F_{0}(x)\right|,\left|G_{0}(x)\right|\right)$.

The following lemmas yield estimates for the derivative of the functions $f_{1}\left(x+\lambda_{1} y\right)$ and $g_{1}\left(x-\lambda_{1} y\right)$. This is accomplished by noticing that if $u(x, y)$ satisfies (2.1), then $u_{x}(x, y)$ also satisfies the same equation. By observing the nature of the boundary values of $u_{x}(x, y)$ we can employ the above lemmas to estimate the functions $f_{1}^{\prime}\left(x+\lambda_{1} y\right)$ and $g_{1}^{\prime}:\left(x-\lambda_{1} y\right)$.

Lemma 2.4. Let the hypotheses of Lemma 2.1 hold and let $F_{0}(x), 0 \leqq x \leqq 2$, be twice differentiable. Then, in the strip $y_{1} \leqq y \leqq 0, f_{1}^{\prime}\left(x+\lambda_{1} y\right)$ and $g_{1}^{\prime}:\left(x-\lambda_{1} y\right)$ satisfy the inequalities

$$
\left|f_{1}^{\prime}\right| \leqq \frac{2 \lambda_{m}}{\lambda_{1}} M^{\prime}, \quad\left|g_{1}^{\prime}\right| \leqq \frac{2 \lambda_{m}}{\lambda_{1}} M^{\prime},
$$

$M^{\prime}=\max \left|F_{0}^{\prime}:(x)\right|, 0 \leqq x \leqq 2$.

Proof. By hypothesis $u(x, 0)=F_{0}(x), 0 \leqq x \leqq 2$, where $u(x, y)$ is the solution given by Lemma 2.1. Hence we have $u_{x}(x, 0)=F_{0}^{\prime}(x), 0 \leqq x \leqq 2$. Along the characteristic $h_{2}(x)$ we have the relation $u_{x}\left[x, h_{2}(x)\right]=g_{1}^{\prime}(2), 1 \leqq x \leqq 2$. To see this we note that $u_{x}(x, 0)=f_{1}^{\prime}(x)+g_{1}^{\prime}(x)$. However (see Fig. 1) we have $f_{1}^{\prime}(x)=0$ in $A_{1}$ and hence $u_{x}(2,0)=g_{1}^{\prime}(2)$. Therefore along $P_{0} P_{1}, u_{x}$ $=g_{1}^{\prime}(2)=$ const. At $P_{1}$ we have $g_{2}^{\prime}=g_{1}^{\prime}$ since both $f_{1}^{\prime}\left(x+\lambda_{1} y\right)$ and $f_{2}^{\prime}\left(x+\lambda_{2} y\right)$ vanish at $P_{1}$. Thus $u_{x}\left[x, h_{2}(x)\right]=$ const. $=g_{1}^{\prime}(2), 1 \leqq x \leqq 2$. Since $u(x, y)$ satisfies the conditions of Lemma 2.1 and $F_{0}(x)$ is twice differentiable, $u_{x}(x, y)$ will satisfy the conditions of Lemma 2.3 with the boundary values $F_{0}^{\prime}(x)$, $0 \leqq x \leqq 2$, along $y=0$ and with the value $F_{0}^{\prime}(2)$ along the characteristic $h_{2}(x)$. Therefore (2.12) holds.

Lemma 2.5. Let the hypotheses of Lemma 2.2 hold and let $G_{0}(x), 1 \leqq x \leqq 2$, be twice differentiable. Then in the strip $y_{1} \leqq y \leqq 0, f_{1}^{\prime}\left(x+\lambda_{1} y\right)$ and $g_{1}^{\prime}\left(x-\lambda_{1} y\right)$ satisfy the inequalities

$$
\left|f_{1}^{\prime}\right| \leqq \frac{\lambda_{m}}{\lambda_{1}} M^{\prime}, \quad\left|g_{1}^{\prime}\right| \leqq \frac{\lambda_{m}}{\lambda_{1}} M^{\prime},
$$

$M^{\prime}=\max \left|G_{0}^{\prime}(x)\right|, 1 \leqq x \leqq 2$.

Proof. By hypothesis we have $u(x, 0)=0,0 \leqq x \leqq 2$; hence $u_{x}(x, 0)=0$, 
$0 \leqq x \leqq 2$. Along the characteristic $h_{2}(x)$ we have

$$
u_{x}\left[x, h_{2}(x)\right]=\frac{1}{2} G_{0}^{\prime}(x)-\frac{1}{2} G_{0}^{\prime}(2), \quad 1 \leqq x \leqq 2 .
$$

This last relation may be shown in the following way. In $A_{1}$ (Fig. 1) we have $u_{x}(2,0)=f_{1}^{\prime}(2)+g_{1}^{\prime}(2)=0$. Hence $f_{1}^{\prime},(2)=-g_{1}^{\prime}(2), f_{1}(2 x-2)=G_{0}(x)$, and $f_{1}^{\prime}(2 x-2)=G_{0}^{\prime}(x) / 2$. Since in $A_{1}$ we have $u_{x}(x, y)=f_{1}^{\prime}\left(x+\lambda_{1} y\right)+g_{1}^{\prime}\left(x-\lambda_{1} y\right)$ and $u_{x}\left[x, h_{2}(x)\right]=f_{1}^{\prime}(2 x-2)+g_{1}^{\prime}(2)$, then $u_{x}\left[x, h_{2}(x)\right]=G_{0}^{\prime}(x) / 2-G_{0}^{\prime}(2) / 2$. Along $P_{1} Q_{1}$ we find $f_{1}^{\prime}\left(x+\lambda_{1} y_{1}\right)+g_{1}^{\prime}\left(x-\lambda_{1} y_{1}\right)=f_{2}^{\prime}\left(x+\lambda_{2} y_{1}\right)+g_{2}^{\prime}\left(x-\lambda_{2} y_{1}\right)$. In particular, at $P_{1}$ we have $f_{1}^{\prime}\left(2+2 \lambda_{1} y_{1}\right)=G_{0}^{\prime},\left(2+\lambda_{1} y_{1}\right) / 2$ and $f_{2}^{\prime},\left(2+\lambda_{1} y_{1}+\lambda_{2} y_{1}\right)$ $=G_{0}^{\prime}\left(2+\lambda_{1} y_{1}\right) / 2$. Thus $g_{2}^{\prime},\left(2+\lambda_{1} y_{1}-\lambda_{2} y_{1}\right)=g_{1}^{\prime}$.(2) and we see that (2.14) holds along $P_{1} P_{2}$. Continuing in this way we see that (2.14) holds along the entire characteristic $h_{2}(x)$. Therefore $u_{x}(x, y)$ satisfies the hypotheses of Lemma 2.2 and we get the inequalities (2.13).

With the aid of the results of Bers for the Cauchy problem for equation (1.1) the above lemmas yield bounds for the solution $u(x, y)$ in the domain $D(0,2)$. We first state a theorem of Bers [2, p. 31].

THEOREM 2.1. Let $\tau(x)$ and $\nu(x)$ be twice continuously differentiable functions defined for $0 \leqq x \leqq 2$. Then there exists a unique solution $u(x, y)$ of equation (1.1) in $D(0,2)$ satisfying the initial conditions $u(x, 0)=\tau(x), u_{y}(x, 0)=\nu(x)$, $0 \leqq x \leqq 2$. In $D(0,2), u(x, y)$ satisfies the inequalities

$$
|u(x, y)| \leqq T+|y| N, \quad\left|u_{y}(x, y)\right| \leqq A T^{\prime}+B N^{\prime}
$$

where $A=A(y)=(-K(y))^{1 / 2}, B=B(x, y)=x-a+|y| A(y), T=\max |\tau(x)|$, and $N=\max |\nu(x)|, T^{\prime}=\max \left|\tau^{\prime}(x)\right|, N^{\prime}=\max \left|\nu^{\prime}(x)\right|$.

Theorem 2.1 applies equally well to equation (2.1).

LеммA 2.6. Let the hypotheses of Lemma 2.3 hold, and suppose that $F_{0}(x)$ and $G_{0}(x)$ possess two continuous derivatives. Then in $D(0,2)$ the solution $u(x, y)$ of equation (2.1) satisfies the inequality

$$
|u(x, y)| \leqq M_{0}+4 \lambda_{m}|y| M_{1},
$$

where $M_{0}=\max \left(\left|F_{0}(x)\right|,\left|G_{0}(x)\right|\right)$ and $M_{1}=\max \left(\left|F_{0}^{\prime}(x)\right|,\left|G_{0}^{\prime}(x)\right|\right)$.

Proof. From the lemmas already proved we have in the strip $y_{1} \leqq y \leqq 0$

$$
\begin{array}{ll}
\left|f_{1}\left(x+\lambda_{1} y\right)\right| \leqq \frac{2 \lambda_{m}}{\lambda_{1}} M_{0}, & \left|g_{1}\left(x-\lambda_{1} y\right)\right| \leqq \frac{2 \lambda_{m}}{\lambda_{1}} M_{0}, \\
\left|f_{1}^{\prime}\left(x+\lambda_{1} y\right)\right| \leqq \frac{2 \lambda_{m}}{\lambda_{1}} M_{1}, & \left|g_{1}^{\prime}\left(x-\lambda_{1} y\right)\right| \leqq \frac{2 \lambda_{m}}{\lambda_{1}} M_{1} .
\end{array}
$$

Differentiation with respect to $y$ in the first strip yields

$$
u_{y}(x, y)=\lambda_{1} f_{1}^{\prime}\left(x+\lambda_{1} y\right)-\lambda_{1} g_{1}^{\prime}\left(x-\lambda_{1} y\right) .
$$


Thus, for $y=0$

$$
\left|u_{y}(x, 0)\right| \leqq \lambda_{1}\left|f_{1}^{\prime}(x)\right|+\lambda_{1}\left|g_{1}^{\prime}(x)\right| \leqq 4 \lambda_{m} M_{1} .
$$

Employing Theorem 2.1, we obtain (2.15) immediately.

3. The general case. To prove Theorem 1.1 we consider, in place of equation (1.1), the system of equations

$$
v_{x}=u_{y}, \quad v_{y}=-K(y) u_{x} .
$$

It is clear that if $u(x, y)$ is a solution of (3.1) which possesses continuous partial derivatives of the second order throughout $D(0,2)$, then $u(x, y)$ will also satisfy equation (1.1). The system (3.1) implies that

$$
\int_{C} v d x-K u d y=0, \quad \int_{C} u d x+v d y=0
$$

for every closed curve $C$ in $D(0,2)$.

LEMma 3.1. Let $K(y), c \leqq y \leqq 0$, be a continuous nondecreasing function of $y$. Let $K_{n}(y)$ be a sequence of negative nondecreasing step-functions, each with a finite number of steps, which converges uniformly to $K(y), c \leqq y \leqq 0$. Let $F_{0}(x)$ and $G_{0}(x)$ be functions with continuous third derivatives. Let $u_{n}(x, y)$ be the solution of

$$
K_{n}(y) u_{x x}+u_{y y}=0
$$

satisfying the boundary conditions $u_{n}(x, 0)=F_{0}(x), 0 \leqq x \leqq 2, u_{n}\left[x, h_{n}(x)\right]$ $=G_{0}(x), 1 \leqq x \leqq 2$, where $y=h_{n}(x)$ is the equation of the characteristic of positive slope passing through the point $(2,0)$. Then

$$
\left\{\frac{\partial}{\partial y} u_{n}(x, y)\right\}_{y=0}, \quad n=1,2, \cdots,
$$

is an equicontinuous sequence of functions.

Proof. Set $J_{n}(x)=\left\{\partial u_{n}(x, y) / \partial y\right\}_{y=0}$. From Lemma 2.6 we have $\left|J_{n}(x)\right|$ $\leqq 4 \max \left|K_{n}(y)\right| M_{1}, M_{1}=\max \left(\left|F_{0}^{\prime}(x)\right|,\left|G_{0}^{\prime}(x)\right|\right)$. Since $\partial u_{n}(x, y) / \partial x$ satisfies (3.3) we have, by applying Lemma 2.6 to the function $\partial u_{n}(x, y) / \partial x$,

$$
\left|\frac{\partial}{\partial y}\left[\frac{\partial}{\partial x} u_{n}(x, y)\right]\right|_{y=0} \leqq 4 \max \left|K_{n}(y)\right| \cdot M_{2}
$$

where $M_{2}=\max \left(\left|F_{0}^{\prime \prime}(x)\right|,\left|G_{0}^{\prime \prime}(x)\right|\right)$. However

$$
\left|\frac{\partial}{\partial y}\left[\frac{\partial}{\partial x} u_{n}(x, y)\right]\right|_{y=0}=J_{n}^{\prime}(x)
$$

and hence $\left\{J_{n}(x)\right\}$ and $\left\{J_{n}^{\prime}(x)\right\}$ are uniformly bounded continuous sequences. 
From the equicontinuous sequence of functions obtained in Lemma 3.1 we may select a subsequence $H_{n}(x)$ which converges uniformly to a function $H(x), 0 \leqq x \leqq 2$.

Lemma 3.2. Let $F_{0}(x), H_{n}(x), n=1,2, \cdots$, be continuous functions such that $H_{n}(x) \rightarrow H(x)$ uniformly, $0 \leqq x \leqq 2$. Let $K(y), K_{n}(y)$ be defined as in Lemma 3.1. Then (1) for each $n$ there exists a unique solution $u_{n}(x, y)$ of (3.1) such that $u_{n}(x, 0)=F_{0}(x), \partial u_{n}(x, 0) / \partial y=H_{n}(x)$. (2) The sequence $u_{n}(x, y)$ converges uniformly in $D(0,2)$ to a continuous function $u(x, y)$. (3) $u(x, y)$ satisfies the system (3.1) with the initial conditions $u(x, 0)=F_{0}(x), u_{y}(x, 0)$ $=H(x), 0 \leqq x \leqq 2$. (4) $u(x, y)$ is uniquely determined by (3).

This lemma is a modification of a lemma of Bers [2, p. 38]. The modification occurs in replacing $H(x)$ by a uniformly convergent sequence $H_{n}(x)$. However in the proof given in [2], $H(x)$ is approximated for each $n$ by a polynomial of fixed degree $N$. Hence the approximation of each $H_{n}(x)$ by a polynomial of degree $N$ in such a way that the sequence of polynomials converges uniformly to $H(x)$ allows the use of the same proof as that given by Bers.

Combining Lemmas 2.6, 3.1, and 3.2 we see that, given the two functions $F_{0}(x), G_{0}(x)$ of Theorem 1.1, a function $u(x, y)$ is obtained which satisfies the system (3.1), the conditions $u(x, 0)=F_{0}(x), u_{y}(x, 0)=H(x)$, and the bound (1.2). We still must show that $u(x, y)$ satisfies (1.1), that $u[x, h(x)]$ $=G_{0}(x)$, and that $u(x, y)$ is uniquely determined by (1.1) and the boundary conditions.

Lemma 3.3. Let $F_{0}(x)$ and $G_{0}(x)$ be defined as in Theorem 1.1. Then the function $u(x, y)$ obtained by application of Lemmas 3.1 and 3.2 satisfies equation (1.1).

Proof. From the lemmas of $\S 2$ we note that the functions $\left\{H_{n}(x)\right\}$ possess as many derivatives as $F_{0}(x)$ and $G_{0}(x)$. If we apply Lemma 3.1 for the functions $F_{0}(x)$ and $G_{0}(x)$ we shall obtain, by selecting a subsequence of the $H_{n}(x)$, a sequence, say $L_{n}(x)$, such that it and its first two derivatives converge uniformly. We shall obtain a function $U^{*}(x, y)$ which satisfies the system (3.1) and the conditions $U^{*}(x, 0)=F_{0}^{\prime \prime}(x), U_{y}^{*}(x, 0)=H_{0}^{\prime \prime}(x)$. The function $U^{*}(x, y)$ will define, except for a constant, a function $V^{*}(x, y)$. We define the functions

$$
U_{1}^{*}(x, y)=\int^{(x, y)} U^{*} d x+V^{*} d y, \quad V_{1}^{*}(x, y)=\int^{(x, y)} V^{*} d x-K U^{*} d y,
$$

which because of (3.2) are independent of the path. The functions $U_{1}^{*}(x, y)$, $V_{1}^{*}(x, y)$ will again satisfy $(3.1)$ and $U_{1}^{*}(x, y)$ will satisfy the conditions $U_{1}^{*}(x, 0)=F_{0}^{\prime}(x), \partial U_{1}^{*}(x, 0) / \partial y=H^{\prime}(x)$. Further, the functions $U_{1}^{*}, V_{1}^{*}$ will be differentiable. We repeat this process again and obtain a function which is 
twice differentiable and which satisfies the initial values of Lemma 3.2. Hence this function will satisfy (1.1).

To show that the proper boundary values are assumed we first extend the definition of $F_{0}(x), G_{0}(x)$ and their derivatives to a slightly larger interval in such a way that all bounds are at most increased slightly. Thus let $F_{0}(x)$, $G_{0}(x)$ be defined in the intervals $-\epsilon_{0} \leqq x \leqq 2+\epsilon_{0}, 1-\epsilon_{0} \leqq x \leqq 2+\epsilon_{0}$, respectively. Select a sequence $\left\{\epsilon_{n}\right\}$ decreasing monotonically to zero and select the sequence of step-functions $\left\{K_{n}(y)\right\}$ so that, for each $y, K_{n}(y)$ decreases monotonically to $K(y)$. For each $n$ the boundary value problem for the domain $D\left(-\epsilon_{n}, 2+\epsilon_{n}\right)$ can be solved and a function $u_{n}(x, y)$ obtained. A modification of Lemmas 3.1 and 3.2 yields a limit function $u(x, y)$ defined in $D(0,2)$ including the boundary. Since $G_{0}(x)=u_{n}\left[x, h_{n}(x)\right]$, we have

$$
\begin{aligned}
&\left|u[x, h(x)]-G_{0}(x)\right| \leqq\left|u[x, h(x)]-u_{n}[x, h(x)]\right| \\
&+\left|u_{n}[x, h(x)]-u_{n}\left[x, h_{n}(x)\right]\right| .
\end{aligned}
$$

However by the convergence of $u_{n}$ the first term on the right approaches zero while the second term approaches zero because of the convergence of the domains. The left side is independent of $n$ and therefore $u[x, h(x)]$ $=G_{0}(x)$.

To establish the uniqueness we suppose that a solution $u(x, y)$ of (1.1) exists with vanishing boundary values. Then except for a constant the system (3.1) defines a function $v(x, y)$. We define the functions

$$
\begin{aligned}
& U_{1}(x, y)=\int^{(x, y)} u d x+v d y, \\
& V_{1}(x, y)=\int^{(x, y)} v d x-K u d y .
\end{aligned}
$$

We note that $U_{1}(x, 0)=0$. Along the characteristic $h(x)$ we have

$$
\begin{aligned}
d v & =v_{x} d x+v_{y} d y=u_{y} d x-K u_{x} d y \\
& =(-K)^{1 / 2} u_{y} d y+(-K)^{1 / 2} u_{x} d x=(-K)^{1 / 2} d u .
\end{aligned}
$$

Since $u=0$ along $h(x)$, we have $d v=0$ and $v=$ const. We may select this constant as zero, and then $U_{1}$ will have vanishing boundary values. We repeat this process again and obtain a twice differentiable function $U_{2}(x, y)$ with vanishing boundary values. Let $h$ be a negative quantity and consider the domain $D_{h}(0,2)$ bounded by the characteristics through $(0,0)$ and $(2,0)$ and by the line $y=h$. If $u(x, y)$ is a solution, then $U_{2}(x, y)$ has continuous partial derivatives with respect to $x$ of the fourth order. Consider the boundary value problem for equation (1.1) in the domain $D_{h}(0,2)$ with the boundary values: zero along the right-hand characteristic and $U_{2}(x, h)$ along the line $y=h$. This problem may be solved by the methods of $\S \S 2$ and 3 and we denote the solu- 
tion thus obtained by $U_{h}^{*}(x, y)$. Since the bounds obtained in $\$ 2$ are independent of the number of strips, we have for the domain $D_{h}(0,2)$

$$
\left|U_{h}^{*}(x, y)\right| \leqq M(1+4 \max |K(y)| \cdot|y|)
$$

where $M=\max \left(\left|U_{2}(x, h)\right|,\left|\partial U_{2}(x, h) / \partial x\right|\right)$. In $D_{h}(0,2)$ equation (1.1) is hyperbolic and the classical uniqueness theorem applies $\left({ }^{2}\right)$. Hence $U_{2}(x, y)$ $=U_{h}^{*}(x, y)$ throughout $D_{h}(0,2)$. Let $\epsilon>0$ be given; we have $\left|U_{2}(x, h)\right|<\epsilon$, $\left|\partial U_{2}(x, h) / \partial x\right|<\epsilon$ for $-h<\delta(\epsilon)$. Thus

$$
\left|U_{2}(x, y)\right| \leqq \epsilon(1+4 \max |K(y) \cdot| y \mid)
$$

throughout $D_{h}(0,2)$. Since $\epsilon$ is arbitrary, $U_{2}(x, y)$ vanishes identically and hence $u(x, y)$ vanishes.

\section{REFERENCES}

1. L. Bers, The Dirichlet problem for a partial differential equation of mixed type, Bull. Amer. Math. Soc. Abstract 54-11-504.

2. - On the continuation of a potential gas flow across the sonic line, N. A. C. A. Technical Note No. 2058, 1950.

3. M. Cibrario, Sui teoremi di existenza e di unicita relativi ad alcune equazioni differenziali a derivate parziali, Rendiconti di R. Accademia dei Lincei (6) vol. 13 (1931).

4. G. Darboux, Lę̧ons sur la thêorie générale des surfaces, vol. 2, Paris, 1889.

5. F. I. Frankl, On the Cauchy problem for equations of mixed elliptic-hyperbolic type with initial data on the parabolic line, Bull. Acad. Sci. URSS. vol. 8 (1944).

6. S. Gellerstedt, Quelques problèmes mixtes pour l'équation $y^{m} z_{x x}+z_{y y}=0$, Arkiv för Matematik Astronomie och Fysik vol. 26 (1937). (References to Gellerstedt's previous papers are given here.)

7. P. Germain and R. Bader, Sur quelques problemes aux limites, singuliers, pour une équation hyperbolique, C. R. Acad. Sci. Paris vol. 231 (1950) p. 268.

8. F. Tricomi, Sulle equazioni lineari alle derivate parziali di $2^{\circ}$ ordine, di tipo misto, Atti della Accademia Nazionali dei Lincei (5) vol. 14 (1923).

The Institute for Advanced Study,

PRinceton, N. J.

SyraCUSE UNIVERSITY,

Syracuse, N. Y.

(2) See, for example, E. Goursat, Cours d'analyse mathematique, 4th ed., Paris, 1927, vol. 3, p. 119. 